\title{
Structural Response of a Reciprocating Compressor's Discharge Tube Subjected to Model and Data Uncertainties
}

\author{
Filipe Fontanela, Olavo M. Silva, Thiago Fiorentin and Arcanjo Lenzi \\ Deptartment of Mechanical Engineering, Universidade Federal de Santa Catarina, Florianópolis - SC, 88040-900, \\ Brasil.
}

(Received 25 May 2017; accepted 2 March 2018)

\begin{abstract}
The analysis of the dynamical responses of compressor components are typically evaluated by using mathematicalmechanical models, and many decisions are given based on numerical simulations. Such an investigation is usually performed in a deterministic framework that cannot consider the uncertainties of the numerical model. These uncertainties are present in a numerical investigation due to the variability of the model parameters, caused by the limitations of the manufacturing processes, as well as simplifications and/or lack of knowledge to describe complex physical processes accurately. In order to quantify the sensitivity of the model parameters and the epistemic uncertainties of a discharge tube's structural numerical response - solved by the finite element method-two stochastic models are constructed, and their results are simultaneously analysed. The dynamical responses obtained from both stochastic models identify the robustness limits of the structural response when it is subjected to parameter uncertainties as well as model sensitivity by separating each contribution in the estimated dynamical structural response.
\end{abstract}

\section{INTRODUCTION}

In structural dynamics, a mathematical-mechanical model is constructed to quantify the physical responses of real structures. The first source of uncertainties during the modelling processes, called data or parameter ${ }^{1,2}$ uncertainties, is attributed to the variability of manufacturing processes. This sort of uncertainty affects model parameters like geometry, mechanical properties, and boundary conditions, among others. The second source of uncertainties, called model or epistemic uncertainties, ${ }^{1,3}$ is attributed mainly to assumed simplifications and/or the lack of knowledge to describe complex physical processes accurately. It is important to consider uncertainties during a modelling process as doing so can improve model predictability, ${ }^{2}$ especially at high frequencies at which small variations in system configuration can lead to very different structural responses. ${ }^{4}$

The main purpose of this paper is to analyse a structural response of a discharge tube used in household compressors when subjected to data and model uncertainties. Figure 1 displays a scheme of a typical hermetic compressor used in household refrigerators. It is mainly composed of a compression mechanism, which generates high impulsive forces due to valve opening or closing, and a compressor housing, which is the most prominent source of noise in a household refrigerator. ${ }^{5}$ In order to decrease the vibration energy transmissibility from the compression mechanism to the housing, and consequently decrease the global compressor noise, the compression mechanism is suspended by springs. The spring stiffness values are selected to attain a mass-spring system with natural frequencies much lower than the operational frequencies of the compressor. The refrigerant fluid is conducted from the compression mechanism to the refrigeration system by means of the discharge tube. The tube serve as the main vibration energy path from the compression mechanism to the housing,

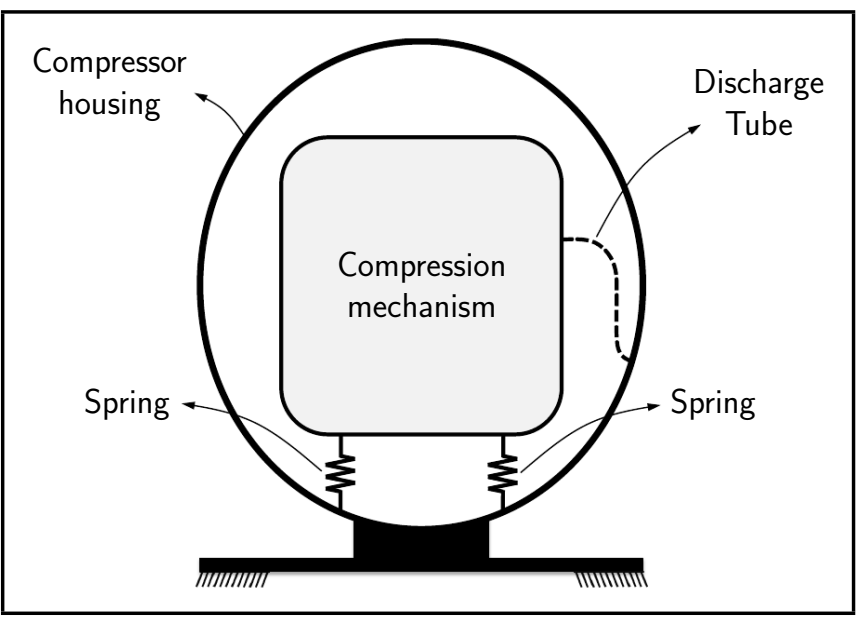

Figure 1. Scheme defining a typical household compressor.

and due its structural importance, it is designed with a particular geometry to decrease energy transmissibility from the former to the latter. However, due to its low dynamical stiffness, the first natural frequencies of the discharge tube are very close to the operational values. ${ }^{6,7}$ Thus any small variation in the structural configuration can lead to system resonance.

In order to investigate the sensitivity of a discharge tube finite element model's sensitivity to data and model uncertainties, two stochastic models are constructed based on different formulations for random uncertainties modelling. The first one, called parametric probabilistic approach, allows the consideration of data uncertainties, modelling every uncertain parameter as a random variable. This is a very efficient tool to quantify data uncertainties; however, by definition, it cannot take model uncertainties into account. ${ }^{8}$ In order to investigate model sensitivity, a nonparametric probabilistic approach based on the maximum entropy principle and the random matrix theory is applied. ${ }^{9}$ This is a very efficient tool to evaluate 


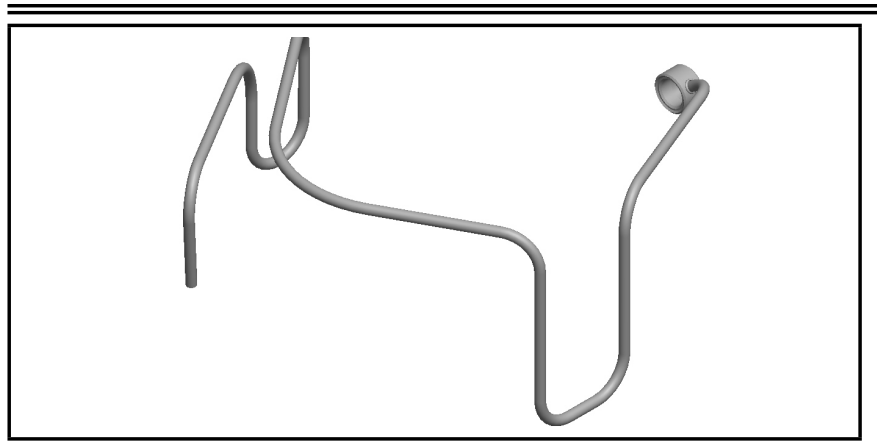

Figure 2. Isometric view of the analysed discharge tube.

both model and data uncertainties and, when applied together with a parametric probabilistic approach, it can estimate the robustness of system responses to model uncertainties. ${ }^{10}$

Section 2, presents the mean computational finite element model constructed to investigate a structural response of the discharge tube. Section 3 is devoted to the construction of parametric and nonparametric probabilistic models of random uncertainties and presents the methodology used to set the same level of uncertainty in each stochastic model. Finally, Section 4 presents and discusses the dynamical responses calculated using the stochastic models and identifies the robustness limits of structural responses to parameter variabilities and model uncertainties.

\section{MEAN DISCHARGE TUBE MODEL}

Figure 2 displays an isometric view of the discharge tube. In order to investigate the dynamical responses of the structure, its geometry is discretized using the finite element method. The nominal model is tested using a commercial code that describes each element as an Euler-Bernoulli beam with two nodes and six degrees of freedom in each node with three translational displacements along the nodal $x, y$ and $z$ directions and three rotational motions about the nodal $x, y$ and $z$ directions. The tube is made of coppered steel, with Young's modulus $E=200 \mathrm{GPa}$, Poisson's ratio $\nu=0.3$, mass density $\rho=7870 \mathrm{~kg} / \mathrm{m}^{3}$, external diameter $\phi=3.2 \mathrm{~mm}$, and wall thickness $s=0.5 \mathrm{~mm}$. The connector coupled with the pipe is modelled as a point mass of $m_{c}=4.25 \mathrm{~g}$. Include the connector in the analysis is very important because its mass is approximately $20 \%$ of the entire discharge tube mass. The structure is clamped in (a) and free in (b), where it is excited by applying a unitary point force in the $z$ direction.

The linear time-invariant damped system modelled with the finite element method can be written in the frequency domain as:

$$
\left(-\omega^{2}\left[M_{n}\right]+j \omega\left[D_{n}\right]+\left[K_{n}\right]\right)\left\{u_{n}(\omega)\right\}=\left\{f_{n}(\omega)\right\} ;
$$

where $\omega$ is the angular frequency, $\left\{f_{n}(\omega)\right\}$ is the vector with $n$ inputs, $\left\{u_{n}(\omega)\right\}$ is the vector with $n$ degrees of freedom, and $j$ is the imaginary unit. In Eq. (1), the mass $\left[M_{n}\right]$, damping $\left[D_{n}\right]$, and stiffness $\left[K_{n}\right]$ operators are symmetric positivedefinite matrices. The vector $\left\{u_{n}(\omega)\right\}$ of the system can be calculated by solving:

$$
\left\{u_{n}(\omega)\right\}=\left(-\omega^{2}\left[M_{n}\right]+j \omega\left[D_{n}\right]+\left[K_{n}\right]\right)^{-1}\left\{f_{n}(\omega)\right\} .
$$

We are interested in studying the structural acceleration vector $\left\{a_{n}(\omega)\right\}$ due to the external point force. This vector can be found using $\left\{u_{n}(\omega)\right\}$, following the relation:

$$
\left\{a_{n}(\omega)\right\}=-\omega^{2}\left\{u_{n}(\omega)\right\}
$$

\subsection{Mean discharge tube reduced model}

In order to decrease the number of algebraic operations using $\left[M_{n}\right],\left[D_{n}\right]$, and $\left[K_{n}\right]$ (and, consequently, decreasing the computational efforts), the mean reduced model is constructed from the usual modal analysis. The generalized eigenvalue problem is written as:

$$
\left[K_{n}\right]\left\{\phi_{i}\right\}=\omega_{i}^{2}\left[M_{n}\right]\left\{\phi_{i}\right\}
$$

where $\omega_{i}$ is the $i$-th eigenvalue and $\left\{\phi_{i}\right\}$ is its corresponding eigenvector. Defining the matrix $\left[\Phi_{m}\right]=\left[\left\{\phi_{1}\right\}\left\{\phi_{2}\right\} \ldots\left\{\phi_{m}\right\}\right]$ with $m \ll n$, the system response $\left\{u_{n}(\omega)\right\}$ can be approximated by $\left\{u_{m}(\omega)\right\}$, such that:

$$
\left\{u_{m}(\omega)\right\}=\left[\Phi_{m}\right]\left\{q_{m}(\omega)\right\} ;
$$

where $\left\{q_{m}(\omega)\right\}$ is the vector solution of:

$$
\begin{gathered}
\left(-\omega^{2}\left[M_{m}\right]+j \omega\left[D_{m}\right]+\left[K_{m}\right]\right)\left\{u_{m}(\omega)\right\}= \\
{\left[\Phi_{m}\right]\left\{f_{n}(\omega)\right\} .}
\end{gathered}
$$

In Eq. (6), the diagonal reduced matrices $\left[M_{m}\right],\left[D_{m}\right]$, and $\left[K_{m}\right]$ can be represented algebraically, assuming the normalization $\left[\Phi_{m}\right]^{T}\left[M_{n}\right]\left[\Phi_{m}\right]=\left[I_{m}\right]$, where $\left[I_{m}\right]$ is the identity matrix, as:

$$
\left[M_{m}\right]=\delta_{i j},\left[D_{m}\right]=2 \xi_{i} \omega_{i} \delta_{i j}, \quad\left[K_{m}\right]=\omega_{i}^{2} \delta_{i j}
$$

where $\delta_{i j}$ is Kronecker's delta, and $\xi_{1}=\xi_{2}=\ldots=\xi_{m}=$ $0.25 \%$ are the usual modal damping rates. According to Eq. (3), the approximated acceleration response $\left\{a_{m}(\omega)\right\}$ can be written using the modal solution, such that $\left\{a_{m}(\omega)\right\}=$ $-\omega^{2}\left\{u_{m}(\omega)\right\}$. The error associated with the approximation of the acceleration vector $\left\{a_{n}(\omega)\right\}$ by $\left\{a_{m}(\omega)\right\}$ can be controlled, and it should be reduced, as it is necessary to set the optimal value of $m$, wherein $\left\|\left\{a_{n}(\omega)\right\}-\left\{a_{m}(\omega)\right\}\right\|$ is sufficiently close to zero.

\subsection{Mesh convergence}

In order to check the mesh convergence, the structural natural frequencies are calculated as a function of the number of elements $N_{e}$ used in the finite element model. For the precision to exceed $99.75 \%$ in the $100^{\text {th }}$ natural mode, it is necessary to use a mesh with approximately 300 elements. Figure 3 shows the acceleration spectrum $\left\{a_{m}^{(b)}(\omega)\right\}$ at point (b) (see Fig. 2) in the $z$ direction, which is calculated using the mean reduced model with $m=100$ modes.

\section{STOCHASTIC MODELS}

This section deals with the construction of two different stochastic models of uncertainties. The first, the parametric probabilistic model, considers mechanical and geometrical properties as random variables. The second model, the nonparametric probabilistic model, considers uncertainties at the operator's level. The main differences between the parametric and nonparametric approaches when applied to a very simple dynamical system can be found in Sampaio and Cataldo's 


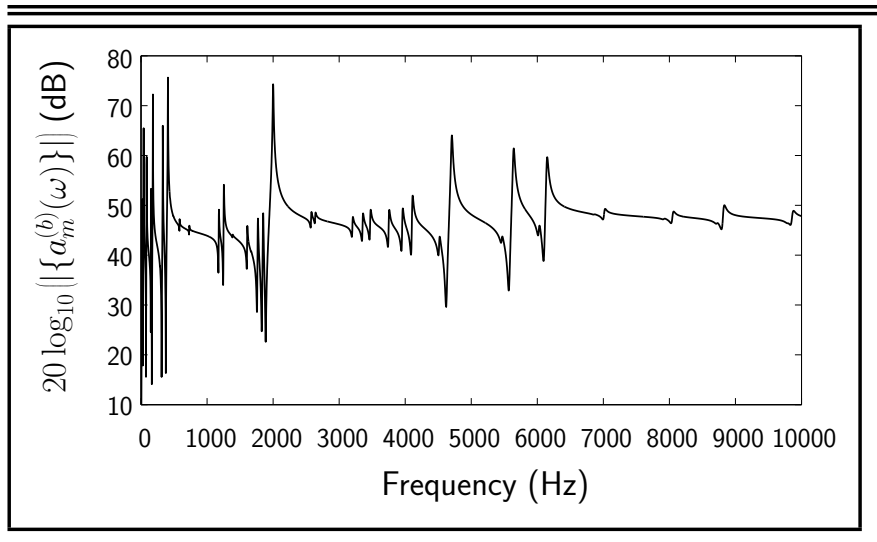

Figure 3. Acceleration spectrum at point (b) in the $z$ direction.

work, ${ }^{12}$ while some physical consequences of using the nonparametric probabilistic approach can be found in the work of Legault et al. ${ }^{4}$ Following the methodology developed by Capiez-Lernout et al., ${ }^{10}$ the nonparametric probabilistic model is fitted to introduce a similar level of uncertainty for each probabilistic model.

\subsection{Parametric probabilistic model}

Let $\{x\}=\left\{p_{1}, p_{2}, \ldots, p_{46}\right\}$ be a vector containing 46 parameters, like curvature radii, inclination angle, Young's modulus, damping ratio, connector mass, and other features of the discharge tube. Therefore, the matrices of Eq. (1) are functions of $\{x\}$. During the parametric probabilistic modelling, vector $\{x\}$ is modelled as a random vector $\{\mathbf{X}\}$ in which the random elements $\mathbf{P}_{\mathbf{i}}$ are independent Gaussian random variables with mean $\mu_{i}$ and standard deviation $\sigma_{i}$. The normal distribution is defined from $-\infty$ to $\infty$; consequently, a Gaussian variable can assume negative values. This behaviour is not acceptable for many necessarily positive parameters of the stochastic model, like Young's modulus, the damping ratio, and the connector mass. However, as the purpose of this paper is to evaluate the sensitivity of the discharge tube model under low levels of uncertainties and, consequently, the probability of the necessarily positive random variables, one may assume negative values can be neglected. The stochastic finite element model is then written as:

$$
\left(-\omega^{2}\left[\mathbf{M}_{\mathbf{n}}^{\text {par }}\right]+j \omega\left[\mathbf{D}_{\mathbf{n}}^{\text {par }}\right]+\left[\mathbf{K}_{\mathbf{n}}^{\text {par }}\right]\right)\left\{\mathbf{U}_{\mathbf{n}}^{\text {par }}\right\}=\left\{f_{n}(\omega)\right\}
$$

where $\left[\mathbf{M}_{\mathbf{n}}^{\text {par }}\right],\left[\mathbf{D}_{\mathbf{n}}^{\text {par }}\right]$, and $\left[\mathbf{K}_{\mathbf{n}}^{\text {par }}\right]$ are the random matrices obtained from the probabilistic model of $\{\mathbf{X}\}$, and $\left\{\mathbf{U}_{\mathbf{n}}^{\text {par }}\right\}$ is the corresponding stochastic process of system responses. In Eq. (8), the operators $\left[\mathbf{M}_{\mathbf{n}}^{\text {par }}\right],\left[\mathbf{D}_{\mathbf{n}}^{\text {par }}\right]$, and $\left[\mathbf{K}_{\mathbf{n}}^{\text {par }}\right]$ are all symmetric positive-definite random matrices. The dispersion of the random vector $\{\mathbf{X}\}$ is controlled by its coefficient of variation, which is chosen as $1 \%$ for all random variables $\mathbf{P}_{\mathbf{i}}$, except the inclination angle variables for which the standard deviations are chosen as 1 degree. Figure 4 shows two different meshes randomly obtained with the parametric probabilistic approach. A numerical solver using the direct Monte Carlo simulation $^{2,9}$ is performed to solve Eq. (8) according to the usual modal analysis described in Subsection 2.1.

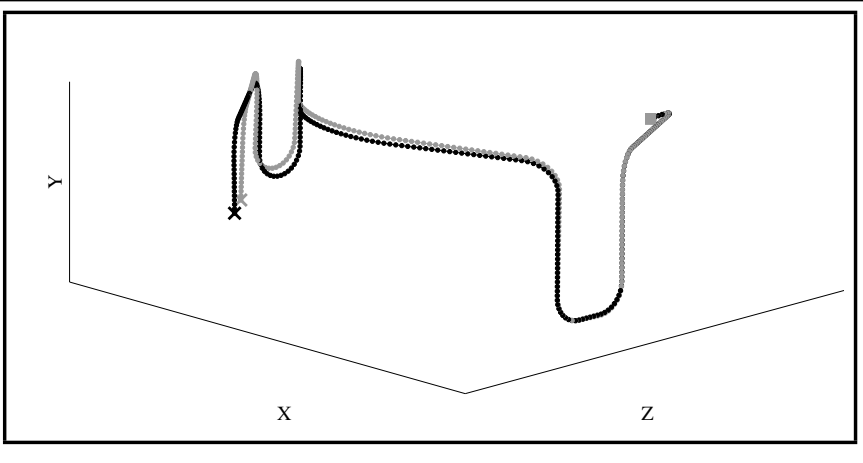

Figure 4. Isometric view of two different meshes randomly obtained using the parametric probabilistic model. The dots represent the node positions, the squares denote the connector point mass, and the crosses show the clamp locations.

\subsection{Nonparametric probabilistic model}

The nonparametric probabilistic modelling process considers uncertainties at the matrix operator's level. The nonparametric approach was proposed by Soize, ${ }^{9}$ and it has been extensively studied in linear ${ }^{13}$ and nonlinear ${ }^{14}$ dynamics, vibroacoustics, ${ }^{15}$ and static ${ }^{16}$ problems. Therefore, the reduced matrix model defined in Eq. (6) is replaced by:

$$
\begin{array}{r}
\left(-\omega^{2}\left[\mathbf{M}_{\mathbf{m}}^{\mathrm{npar}}\right]+j \omega\left[\mathbf{D}_{\mathbf{m}}^{\mathrm{npar}}\right]+\left[\mathbf{K}_{\mathbf{m}}^{\mathrm{npar}}\right]\right)\left\{\mathbf{U}_{\mathbf{m}}^{\text {npar }}\right\}= \\
{\left[\Phi_{m}\right]\left\{f_{n}(\omega)\right\} ;}
\end{array}
$$

where $\left[\mathbf{M}_{\mathbf{m}}^{\mathrm{npar}}\right],\left[\mathbf{D}_{\mathbf{m}}^{\mathrm{npar}}\right]$, and $\left[\mathbf{K}_{\mathbf{m}}^{\mathrm{npar}}\right]$ are symmetric positivedefinite reduced random matrices and $\left\{\mathbf{U}_{\mathbf{n}}^{\text {npar }}\right\}$ is its associated stochastic process. The matrices in Eq. (9) can be rewritten as:

$$
\begin{aligned}
{\left[\mathbf{M}_{\mathbf{m}}^{\mathrm{npar}}\right] } & =\left[L_{M}\right]^{T}\left[\mathbf{G}_{\mathbf{M}}\right]\left[L_{M}\right] ; \\
{\left[\mathbf{D}_{\mathbf{m}}^{\mathrm{npar}}\right] } & =\left[L_{D}\right]^{T}\left[\mathbf{G}_{\mathbf{D}}\right]\left[L_{D}\right] ; \\
{\left[\mathbf{K}_{\mathbf{m}}^{\mathrm{npar}}\right] } & =\left[L_{K}\right]^{T}\left[\mathbf{G}_{\mathbf{K}}\right]\left[L_{K}\right] ;
\end{aligned}
$$

where $\left[L_{M}\right],\left[L_{D}\right]$, and $\left[L_{K}\right]$ are diagonal matrices obtained from the usual Cholesky decompositions $\left[M_{m}\right]=$ $\left[L_{M}\right]^{T}\left[L_{M}\right],\left[D_{m}\right]=\left[L_{D}\right]^{T}\left[L_{D}\right]$, and $\left[K_{m}\right]=\left[L_{K}\right]^{T}\left[L_{K}\right]$. In Eqs. 10,11 , and 12 , the operators $\left[\mathbf{G}_{\mathbf{M}}\right],\left[\mathbf{G}_{\mathbf{D}}\right]$, and $\left[\mathbf{G}_{\mathbf{K}}\right]$ can be modelled as independent positive-definite symmetric random matrices ${ }^{9,11}$ for which probability density functions must be constructed.

The random matrix $[\mathbf{G}]$ denotes $\left[\mathbf{G}_{\mathbf{M}}\right],\left[\mathbf{G}_{\mathbf{D}}\right]$, or $\left[\mathbf{G}_{\mathbf{K}}\right]$. The probability model of $[\mathbf{G}]$ is constructed according to the random matrix theory and the maximum entropy principle using the available informations as constraints. ${ }^{2,9}$ The probability density function of random matrix $[\mathbf{G}]$ with respect to the Lebesgue measure $\tilde{d} G=2^{n(n-1) / 4} \prod_{1 \leq i \leq j \leq n} \mathrm{~d} G_{i j}$ is given by 2,9

$$
\begin{array}{r}
p_{G}([G])=\mathbb{1}_{\mathrm{M}^{+}}([G]) \times C_{G} \times \operatorname{det}([G])^{(m+1) \frac{1-\delta_{G}}{2 \delta_{G}}} \times \\
\exp \left\{-\frac{m+1}{2 \delta_{G}^{2}} \operatorname{tr}([G])\right\}
\end{array}
$$

where $\mathbb{1}_{\mathbb{M}^{+}}([G])$ is equal to 1 if $[G] \in \mathbb{M}^{+}$and is to 0 if $[G] \notin$ $\mathrm{M}^{+} ; \mathrm{M}^{+}$denotes the ensemble of all real symmetric positivedefinite matrices with dimensions $m \times m$. In Eq. (13) $C_{G}$ is a 
positive constant, such that:

$$
C_{G}=\frac{(2 \pi)^{-m(m-1) / 4}\left\{(m+1)\left(\sqrt{2} \delta_{G}^{v}\right)^{-2}\right\}^{m(m+1) /\left(2 \delta_{G}^{2}\right)}}{\prod_{j=1}^{m} \Gamma\left(\frac{m+1}{2 \delta_{G}^{2}}+\frac{1-j}{2}\right)} .
$$

The dispersion parameter $\delta_{G}$ denotes the mean quadratic distance between random matrix $[\mathbf{G}]$ from its mean value $\left[I_{m}\right]$ and assumes the value $0 \leq \delta_{G} \leq(m+1)^{1 / 2} \times(m+5)^{-1 / 2}$. The algebraic representation of $[\mathbf{G}]$ can be constructed according to Cholesky factorization. Thus:

$$
[\mathbf{G}]=\left[\mathbf{L}_{\mathbf{G}}\right]^{T}\left[\mathbf{L}_{\mathbf{G}}\right]
$$

where $\left[\mathbf{L}_{\mathbf{G}}\right]$ is an upper triangular random matrix such that:

1. the matrix elements $\left[\mathbf{L}_{\mathbf{G}}\right]_{i j}$ are independent random variables;

2. for $i<j$, the matrix elements $\left[\mathbf{L}_{\mathbf{G}}\right]_{i j}$ can be written as $\left[\mathbf{L}_{\mathbf{G}}\right]=\frac{\delta_{G}}{\sqrt{m+1}} \mathbf{U}_{\mathbf{i j}}$, where $\mathbf{U}_{\mathbf{i j}}$ is a real Gaussian random variable with zero mean and unit variance;

3. for the diagonal $i=j$, the matrix elements $\left[\mathbf{L}_{\mathbf{G}}\right]_{j j}$ can be written as $\left[\mathbf{L}_{\mathbf{G}}\right]=\frac{\delta_{G}}{\sqrt{m+1}} \sqrt{2 \mathbf{V}_{\mathbf{j} \mathbf{j}}}$, where $\mathbf{V}_{\mathbf{j} \mathbf{j}}$ is a gamma random variable whose probability density function is:

$$
p_{V_{j j}}(v)=\frac{1}{\Gamma\left(\frac{m+1}{2 \delta_{G}^{2}}+\frac{1-j}{2}\right)} v^{(m+1) / 2 \delta_{G}^{2}-(1+j) / 2} \exp \{-v\}
$$

A numerical solver using the direct Monte Carlo method is implemented to solve Eq. (9). The system response is calculated, according to Eq. (5), as a linear combination of the eigenvectors obtained from the mean model.

\subsection{Identification of the matrices' disper- sion parameters with respect to the parametric probabilistic model}

This paper aims to analyse the response sensitivity of an industrial mechanical system to data and model uncertainties. Therefore, it is necessary to set the nonparametric probabilistic model to represent the same levels of uncertainties reproduced by the parametric probabilistic model. The dispersion parameters $\delta_{M}, \delta_{D}$, and $\delta_{K}$ related to the random mass, damping and stiffness matrices, respectively, are then estimated using the methodology developed by Capiez-Lernout et al. ${ }^{10}$ The parametric probabilistic model introduced in Subsection 3.1 includes data uncertainties, while the nonparametric probabilistic model introduced in Subsection 3.2 deals with both model and data uncertainties. An optimization problem is introduced to set the same level of uncertainties in both probabilistic models.

The lowest natural frequencies of the system modelled by the parametric and nonparametric probabilistic approaches are $\Omega_{1}^{\text {par }}$ and $\Omega_{1}^{\text {npar }}$, respectively. The probability density functions $p_{\boldsymbol{\Omega}_{1}^{\text {par }}}$ and $p_{\boldsymbol{\Omega}_{1}^{\text {npar }}}$-related to the random variables $\boldsymbol{\Omega}_{1}^{\text {par }}$ and $\Omega_{1}^{\text {npar }}$, respectively - are compared using the least square cost function: ${ }^{10}$

$$
J\left(\delta_{M}, \delta_{K}\right)=\frac{\left\|p_{\boldsymbol{\Omega}_{1}^{\mathrm{npar}}}-p_{\boldsymbol{\Omega}_{\mathbf{1}}^{\text {par }}}\right\|}{\left\|p_{\boldsymbol{\Omega}_{1}^{\text {par }}}\right\|} ;
$$

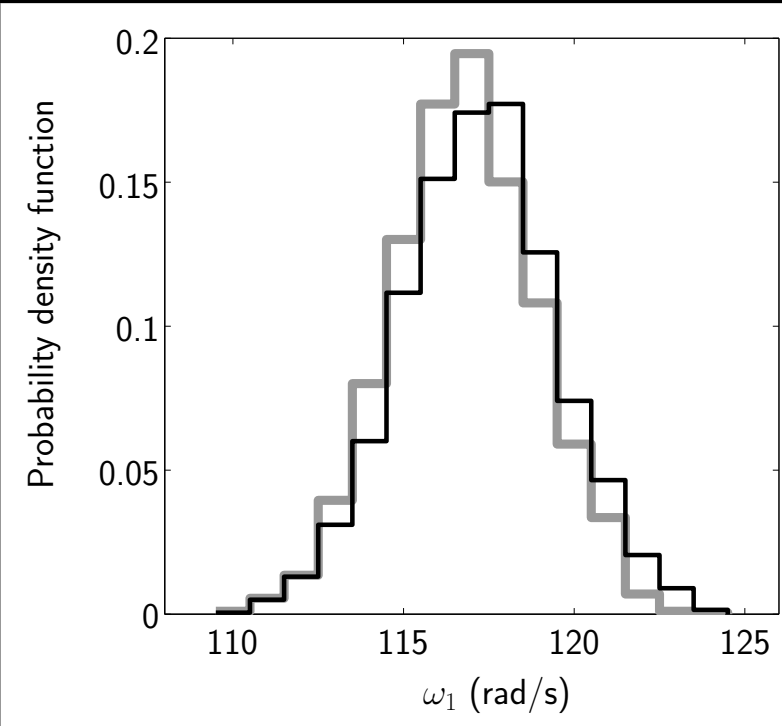

Figure 5. Discrete probability density functions obtained with the parametric (black) and nonparametric (grey) probabilistic approaches.

where $\|f(x)\|$, considering a generic function $f(x)$, is obtained solving $\sqrt{\int_{0}^{\infty}|f(x)|^{2} d x}$. The dispersion parameters $\delta_{M}$ and $\delta_{K}$ are then estimated solving the optimization problem:

$$
\left[\delta_{M}, \delta_{K}\right]=\arg \min J\left(\delta_{M}, \delta_{K}\right)
$$

Since structural natural frequencies are not functions of the damping matrix, the dispersion parameter $\delta_{D}$ cannot be included in the cost function defined by Eq. (17). Therefore, $\delta_{D}$ is determined using the relation: ${ }^{10}$

$$
\delta_{D}=\sqrt{\frac{W_{D}(m+1)}{\operatorname{tr}\left\{\left[D_{m}\right]\right\}^{2}+\operatorname{tr}\left\{\left[D_{m}\right]^{2}\right\}}} ;
$$

where $W_{D}$ is the mean quadratic distance of random matrix $\left[\mathbf{D}_{\mathbf{m}}^{\text {npar }}\right]$ from its mean value. The value of $W_{D}$ can be numerically estimated solving:

$$
W_{D}=\mathcal{E}\left\{\left\|\left[\mathbf{D}_{\mathbf{m}}^{\mathrm{npar}}\right]-\left[D_{m}\right]\right\|_{F}^{2}\right\} .
$$

\section{SIMULATED RESULTS OBTAINED USING THE STOCHASTIC MODELS}

\subsection{Estimation of the dispersion parame- ters}

In order to estimate the best values of $\delta_{M}$ and $\delta_{K}$, the optimization problem defined by Eq. (18) is solved using the genetic algorithm with $n_{s}=2000$, where $n s$ is the number of simulations. The optimization problem yields $\delta_{M}=0.276$ and $\delta_{K}=0.011$. Figure 5 shows $p_{\boldsymbol{\Omega}_{1}^{\text {par }}}$ and $p_{\boldsymbol{\Omega}_{1}^{\text {npar }}}$ using the best estimated values. The best value of $\delta_{D}$ is determined by solving Eq. (19).

The value of as a function of the number of simulations is presented at Fig.ure 6 and, according to the graph, the best value is reached with simulations.

The value of $\delta_{D}$ as a function of the number of simulations $n_{s}$ is presented at Fig. 6 and, according to the graph, the best value $\delta_{D}=0.029$ is reached with $n_{s}=1200$ simulations. 


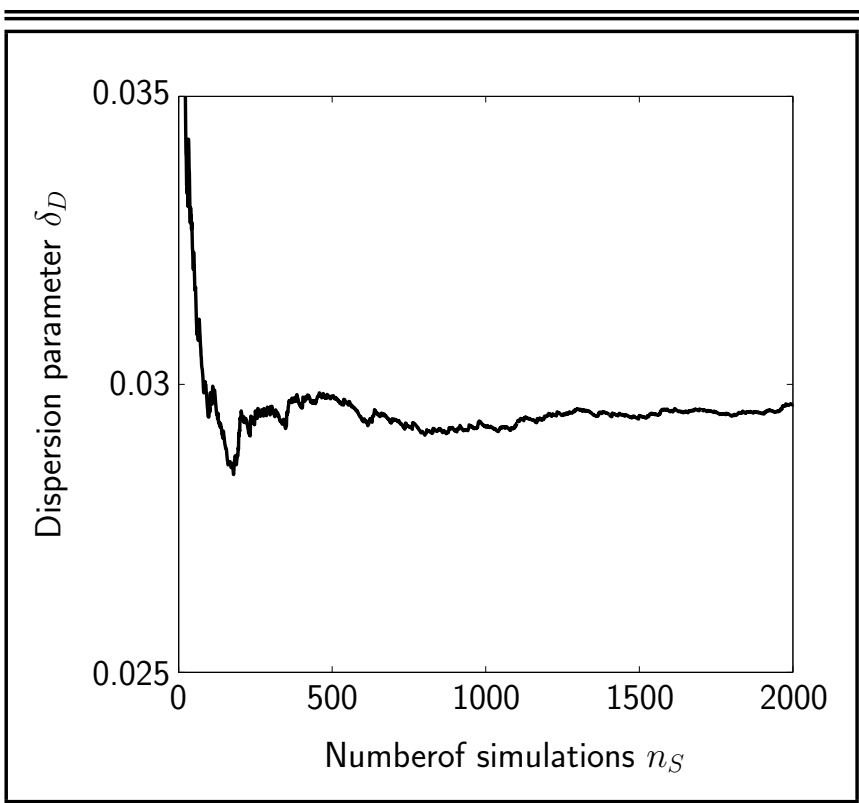

Figure 6. Value of $\delta_{D}$ calculated as a function of $n_{s}$.

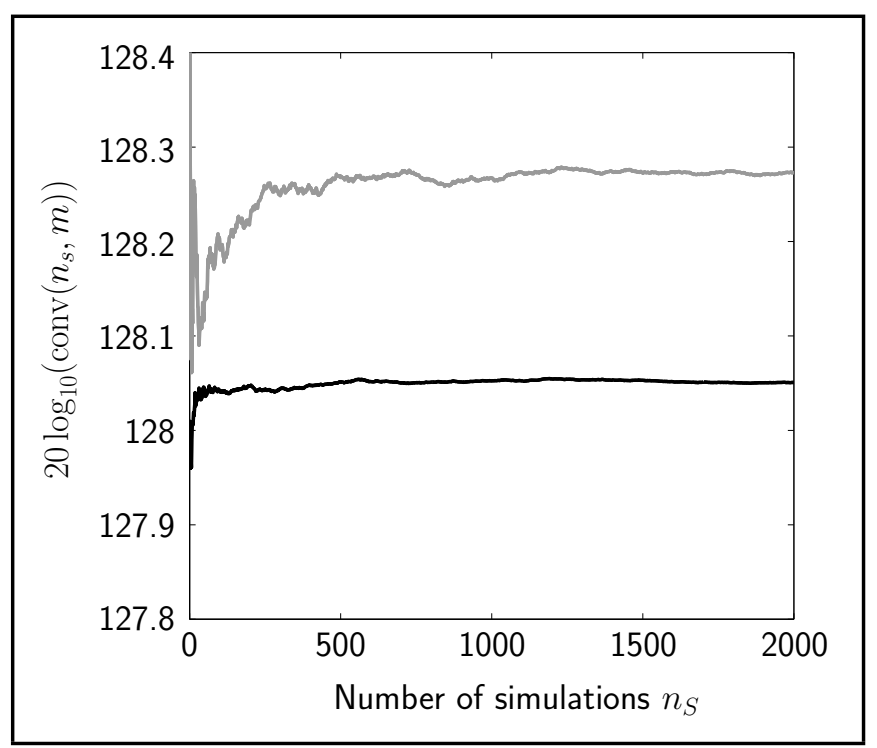

Figure 7. Convergence function calculated using the parametric (black) and nonparametric (grey) models varying $n_{s}$ for $m=100$.

\subsection{Convergence analysis}

In order to set the best values for the number of simulations $n_{s}$ and the dimension $m$ of the reduced model, it is necessary to analyse the results using a convergence criteria. Since we are interested in the connector's random acceleration $\left\{\mathbf{a}_{\mathbf{m}}^{(\mathbf{b})}(\omega)\right\}$, the convergence function is defined as:

$$
\operatorname{conv}\left(n_{s}, m\right)=\left\{\frac{1}{n_{s}} \sum_{k=1}^{n_{s}} \int\left|\left\{\mathbf{a}_{\mathbf{m}}^{(\mathbf{b})}(\omega, k)\right\}\right| d \omega\right\}^{1 / 2} ;
$$

where $k$ denotes the realization index. Figure 7 plots the convergence function by varying $n_{s}$ for $m=100$; according to graph, good convergence is reached with $n_{s}=1400$ simulations. To set the best dimension of the reduced model, the same convergence function is calculated by varying $m$ for $n_{s}=1400$ realizations.

The results are calculated in Fig. 8, and good approximation is obtained using $m=70$ structural modes.

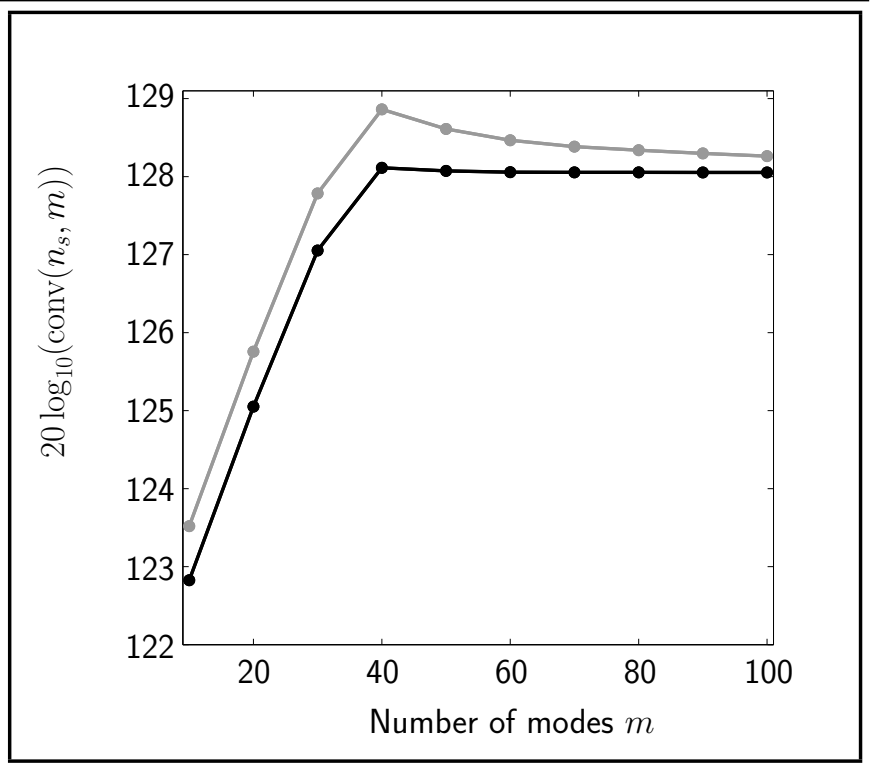

Figure 8. Convergence function obtained using the parametric (black) and nonparametric (grey) models by varying $m$ for $n_{s}=1400$.

\subsection{Random response of the discharge tube}

This section presents the results obtained from the parametric and nonparametric probabilistic models fitted with the same levels of uncertainties. The parametric results are calculated assuming the coefficient of variation for all random variables as $1 \%$, except for the inclination angles, which are assigned a standard deviation of 1 degree. The stochastic response calculated with the nonparametric probabilistic model is obtained using the results $\delta_{M}=0.276, \delta_{D}=0.029$ and $\delta_{K}=0.011$ estimated in Subsection 4.1.

In order to investigate the robustness of the predictions, the acceleration response is divided into three ranges: low frequency $(0-1 \mathrm{kHz})$, medium frequency $(1-5 \mathrm{kHz})$, and high frequency $(5-10 \mathrm{kHz})$. The same finite element mesh is used for all analysis. Figure 9 shows the random responses $\left\{\mathbf{a}_{\mathbf{m}}^{(\mathbf{b})}(\omega, k)\right\}$ in the low frequency range, calculated by applying the parametric (top) and nonparametric (bottom) stochastic models. The grey envelope displays the confidence limits obtained with a probability level of $95 \%$, estimated by applying the sample quantiles method. ${ }^{10,17}$ The resulting graph shows that the random acceleration response appears robust to data uncertainties due to the low variability in the parametric model. The model sensitivity, estimated by the differences between the results of the parametric and nonparametric models, is negligible for frequencies lower than $500 \mathrm{~Hz}$ and starts increasing for frequencies beyond this value.

The random response $\left\{\mathbf{a}_{\mathbf{m}}^{(\mathbf{b})}(\omega, k)\right\}$ in the medium frequency range for the parametric (top) and nonparametric (bottom) models is Fig. 10. The parametric results (top) show that the system is sensitive to data uncertainties, as depicted by the extent of the grey region. The envelope is large, indicating loss of accuracy when predicting the resonance regions themselves. The nonparametric results show that the system is not robust enough to model uncertainties because the grey region in this case is much larger than the one plotted using the parametric model.

The high frequency responses are presented in Fig. 11. The parametric results (top) show that the robustness of the system response toward data uncertainties is small. However, when 


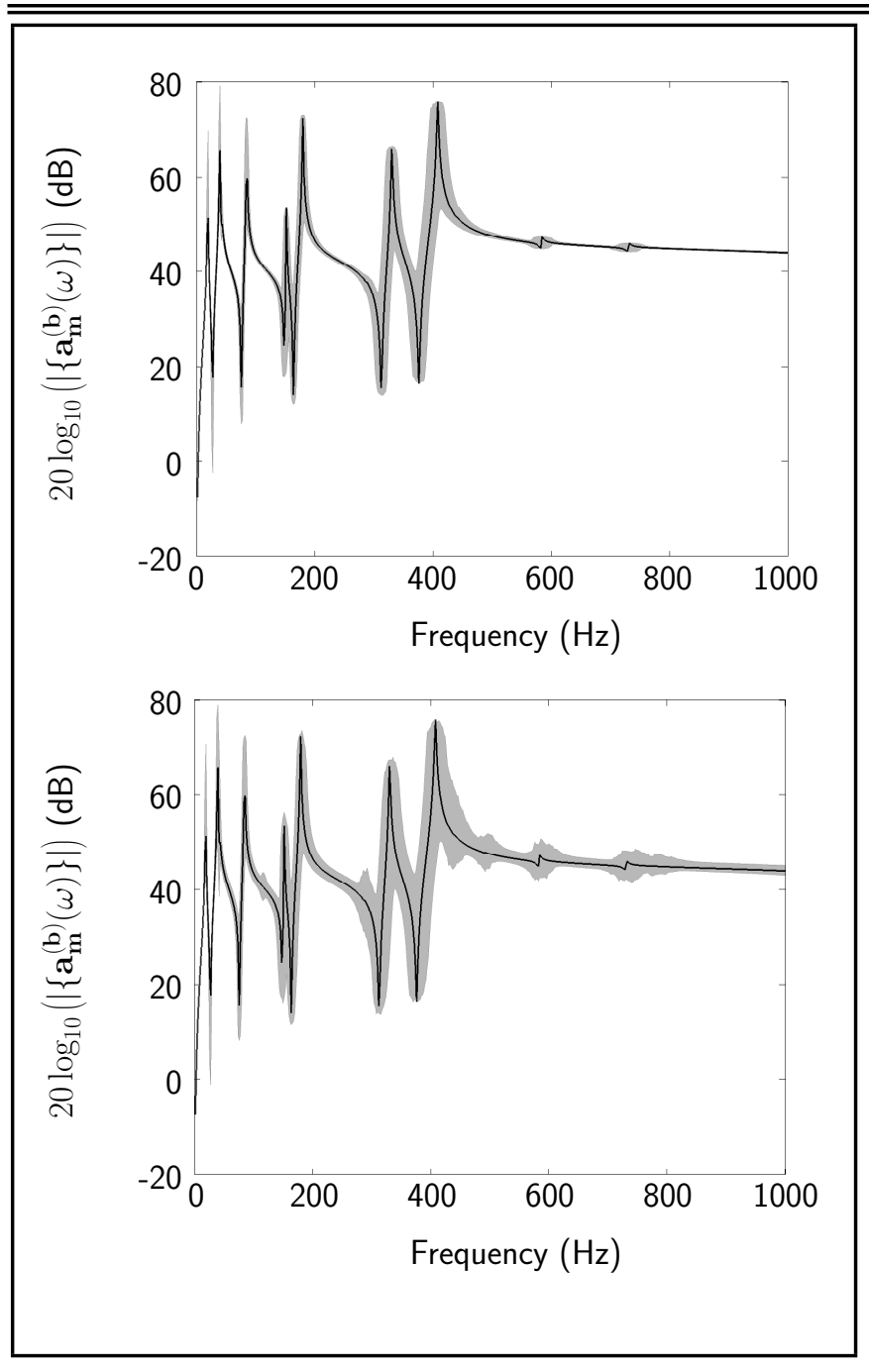

Figure 9. Top: Envelope (grey region) for a probability level of $95 \%$, obtained using the parametric probabilistic model in the low-frequency range. The dark line presents the mean model response. Bottom: The same envelope obtained using the nonparametric probabilistic model.

compared with the nonparametric results (bottom), it is deduced that model uncertainties are more important in this frequency range. This result shows that even a simple dynamic model-like the Euler-Bernoulli mechanical model used to represent the discharge tube in this paper-can be very sensitive to model uncertainties in medium and high frequency ranges. Moreover, decisions based on a deterministic numerical investigation for discharge tube response can lead to misleading results for medium and high frequencies.

\section{CONCLUSIONS}

This paper analysed two different stochastic models of uncertainties applied to a discharge tube used in household refrigerators. The first stochastic model-the parametric probabilistic model—replaced every uncertain parameter by a random variable. This is a very efficient tool to evaluate data uncertainties but, by definition, it cannot consider model uncertainties. The second stochastic model-the nonparametric probabilistic model—can consider both data and epistemic uncertainties. Therefore, when both stochastic models of uncertainties are applied to a structural analysis, it is possible to separate the robustness of responses of a system subjected to both uncertainties.
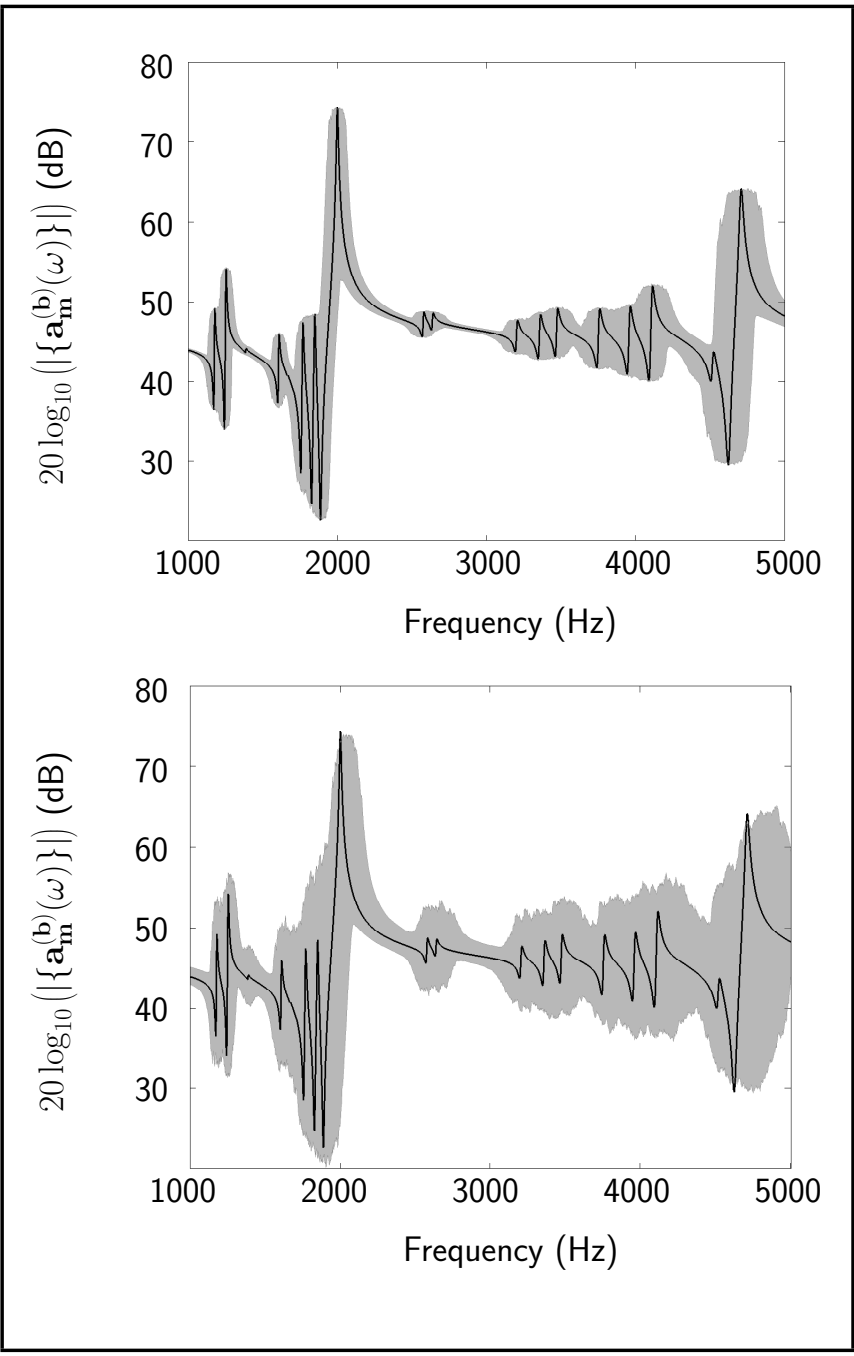

Figure 10. Top: Envelope (grey region) for a probability level of 95\%, obtained using the parametric probabilistic model in the middle-frequency range. The dark line presents the mean model response. Bottom: The same envelope obtained using the nonparametric probabilistic model.

The results obtained in this paper showed that the system response is very robust to data and model uncertainties in the low frequency range. This property is very important because the first natural frequencies of the structure are very close to the operational frequencies. Therefore, decisions based on a deterministic numerical analysis are predictive in the low frequency range. The stochastic structural results for the medium frequency range showed that both the data and the model uncertainties increase, diminishing the robustness of the structural response in this frequency range. In the high frequency range, the robustness of the structural response with respect to data uncertainties continued to be small, but model sensitivity was predominant. This result suggests that a deterministic strategy in this frequency range can lead to erroneous results. Moreover, the results showed that even a very simple industrial component model, like the discharge tube modelled in this study, can be very sensitive to model uncertainties in medium and high frequency ranges.

\section{ACKNOWLEDGEMENTS}

The generous financial support of CNPq - Conselho Nacional de Desenvolvimento Cientifico e Tecnologico is highly appreciated. 


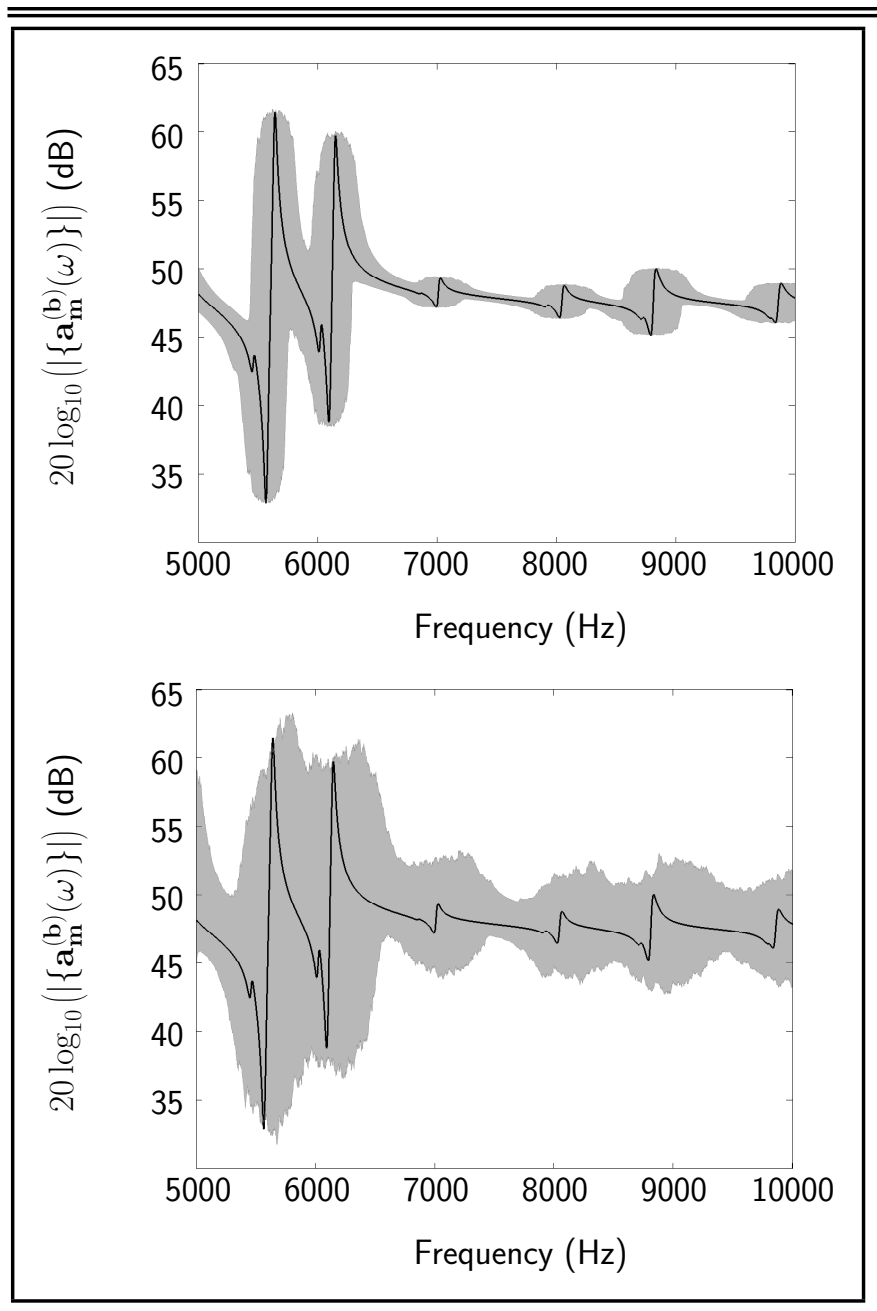

Figure 11. Top: Envelope (grey region) for a probability level of $95 \%$, obtained using the parametric probabilistic model in the high-frequency range. The dark line presents the mean model response. Bottom: The same envelope obtained using the nonparametric probabilistic model.

\section{REFERENCES}

1 C. Soize, Stochastic modeling of uncertainties in computational structural dynamics - Recent theoretical advances, Journal of Sound and Vibration, 332(10), 23792395 (2013) https://dx.doi.org/10.1016/j.jsv.2011.10.010

2 Soize, C., A comprehensive overview of a nonparametric probabilistic approach of model uncertainties for predictive models in structural dynamics, Journal of Sound and Vibration, 288(3), 623-652 (2005) https://dx.doi.org/10.1016/j.jsv.2005.07.009

3 Adhikari, S., Wishart random matrices in probabilistic structural mechanics, Journal of Engineering Mechanics, 134(12), 1029-1044 (2008) https://dx.doi.org/10.1061/(ASCE)07339399(2008)134:12(1029)

4 Legault, J., Langley, R.S., Woodhouse, J., Physical consequences of a nonparametric uncertainty model in structural dynamics, Journal of Sound and Vibration, 331(25), 54645487, (2012) https://dx.doi.org/10.1016/j.jsv.2012.07.017

5 Soedel, W., Sound and vibration of positive displacement compressors, 6-8, Taylor \& Francis, Boca Raton (2007)
6 Gaertner, E.L., Robust design applied to manufacturing analysis, Proceedings of the international compressor engineering conference, Purdue, United States, 2008 July 1417, Purdue (2008), 1-8.

7 Bortoli, M.G., Silva, J.C., The application of Monte Carlo method for sensitivity analysis of compressor components, Proceedings of the international compressor engineering conference, Purdue, United States, 2012 July 16-19, Purdue (2012), 1-10.

8 Soize, C., Random matrix theory for modeling uncertainties in computational mechanics, Computer methods in applied mechanics and engineering, 194(12-16) 1333-1366, Academic Press (2005) https://dx.doi.org/10.1016/j.cma.2004.06.038

9 Soize, C., A nonparametric model of random uncertainties for reduced matrix models in structural dynamics, Probabilistic engineering mechanics, 15(3), 277-294 (2000) https://dx.doi.org/10.1016/S0266-8920(99)00028-4

10 Capiez-Lernout, E., Pellissete, M., Pradlwarter, H., Schuller, G.I., Soize, C., Data and model uncertainties in complex aerospace engineering systems, Journal of Sound and Vibration, 295(3-5), 923-938 (2006) https://dx.doi.org/10.1016/j.jsv.2006.01.056

11 Adhikari, S., Matrix variate distributions for probabilistic structural dynamics, AIAA Journal, 45(7), 1748-1762 (2007) https://dx.doi.org/10.2514/1.25512

12 Sampaio, R., Cataldo, E., Comparing two strategies to model uncertainties in structural dynamics, Shock and vibration, 17(2), 171-186 (2010) https://dx.doi.org/10.3233/SAV-2010-0505

13 Chen, C., Duhamel, D., Soize, C., Probabilistic approach for model and data uncertainties and its experimental identification in structural dynamics: Case of composite sandwich panels, Journal of Sound and Vibration, 294(1-2), 64-81 (2006) https://dx.doi.org/10.1016/j.jsv.2005.10.013

14 Murthy, R., Wang, X., Perez, R., Mignolet, M., Richter, L., Uncertainty-based experimental validation of nonlinear reduced order models, Journal of Sound and Vibration, 331(5), 1097-1114 (2012) https://dx.doi.org/10.1016/j.jsv.2011.10.022

15 Durand, J., Soize, C., Gagliardini, L., Structural-acoustic modeling of automotive vehicles in presence of uncertainties and experimental identification and validation The Journal of the Acoustical Society of America, 124(3), 15131525 (2008) https://dx.doi.org/10.1121/1.2953316

16 Soize, C., Nonparametric probabilistic approach of uncertainties for elliptic boundary value problem, International Journal for Numerical Methods in Engineering, 80(6-7), 673-688 (2009) https://dx.doi.org/10.1002/nme.2563

17 Serfling, R. J., Approximation Theorems of Mathematical Statistics, 74-87, John Wiley \& Sons, Maryland (1980) 